Research Article

\title{
COMPARISON OF INTRAOCULAR PRESSURE IN SPARGUE-DAWLEY RATS USING TWO TYPES OF TONOMETERS
}

\author{
Yoon-Jung Choy ${ }^{1}$, Jee-Hyun Choi ${ }^{2}$ \\ Received 30 June 2021, revised 09 November 2021
}

\begin{abstract}
The present study was designed to compare intraocular pressure (IOP) values by two types of tonometers in condition of normotensive and glaucomatous rat model. Glaucomatous eyes were induced by intracameral injections of hyaluronic acid in right eyes of six-week-old male Spargue-Dawley (SD) rats. Normotensive contralateral eyes were left eyes of the SD rats. IOP was measured using a rebound tonometer (Tonolab) and an immersive tonometer (Tonopen ${ }^{\circledR} \mathrm{XL}$ ) about 3:00 pm. The mean IOP values of normotensive control eyes were $10.80 \pm 1.03 \mathrm{mmHg}$ by Tonopen, and $15.10 \pm 0.73$ mmHg by Tonolab which were statistically insignificant $(p=0.1)$. The mean IOP values of glaucomatous experimental eyes were $30.20 \pm 2.67 \mathrm{mmHg}$ by Tonopen, and $37.90 \pm 2.73 \mathrm{mmHg}$ by Tonolab which were statistically insignificant $(p=0.95)$. High IOP values of glaucomatous eyes by two types of tonometers had strong positive correlation with each other $(r=0.904, p<0.01)$. There was a decrease of ganglion cell layer cells in retina of glaucomatous eye compared to that of normotensive eye. This is the first study to compare IOP values using two types of tonometers between normotensive and glaucomatous model made by intracameral injection of hyaluronic acid. It can be concluded that Tonopen should be used carefully when the IOP is within normal range, and both Tonopen and Tonolab can be used reliably when the IOP is high.
\end{abstract}

Key words: Normotensive, Glaucomatous, Hyaluronic acid, IOP, Rats, Tonolab, Tonopen.

\section{INTRODUCTION}

Glaucoma is one of the most severe ocular diseases, characterized by an irreversible loss of retinal ganglion cells (RGCs) and damage to the optic nerve head (Anders et al. 2018). Elevated intraocular pressure (IOP) is a major risk factor for glaucoma and is characterized by an irreversible decrease in retinal ganglion cells (RGCs) (Choy et al. 2017).

Glaucoma animal model is useful for identifying the etiology of glaucoma and for developing treatment regimens (Morrison et al. 1997, Yu et al. 2007). The glaucoma animal models like dogs and rabbits are expensive (Quigley and Addicks 1980, Quigley and Hohman 1983, Moore et al. 1993) and rabbits suffer from marked differences in optic nerve head structures. Therefore, we sought to develop rat glaucoma model because it is less expensive and is easier to handle.

The aim of this study was to evaluate IOP values measured with two types of tonometers in normal IOP (normotensive) and glaucomatous rat model, and to clarify the relationship and characteristics of both tonometers in practice.

\section{MATERIALS AND METHODS}

\section{Raising environment of SD rats}

Five-week-old male Sprague-Dawley (SD) rats weighing approximately $200 \mathrm{~g}$ and reared by ORIENTBIO were used in this study. All experimental procedures were approved by the Animal Review Board of Eulji University in accordance with the National Institutes of Health Guide for the Care and Use of Laboratory Animals (NIH Publication No. 80-23, revised 1996). The rats were housed in a specific pathogen free laboratory at the Department of Biomedical Laboratory Science at Eulji University in Seongnam. Total 10 SD rats were divided into glaucomatous eyes (right eyes) and normotensive eyes (left eyes). The laboratory environment was maintained at a temperature $20-25^{\circ} \mathrm{C}$, humidity $40-60 \%$, and a 12-hour day/night cycle (7:00 am to 7:00 pm). The rats were freely fed with appropriate

${ }^{1}$ Department of Optometry, Eulji University College of Heath Sciences, Seongnam, Korea, ${ }^{2}$ Laboratory Animal Center, Osong Medical Innovation Foundation, Cheongju, Republic of Korea.

Corresponding author.e-mail: yjchoy7@eulji.ac.kr 
food and allowed to acclimate to their environment for one week prior to beginning the study.

\section{Making rat glaucoma model by intracameral hyaluronic acid injection}

Ten six-week-old male SD rats weighing 250-300 g were anesthetized using an intraperitoneal injection of tiletamine and zolazepam (Zoletil 50 Inj, Virbac Korea, St. Ogeum, Seoul, Korea) and xylazine hydrochloride (Rompun inj, Bayer Korea, St. Boramae, Seoul, Korea) in a $2: 1$ ratio $(0.1 \mathrm{mg} / 100 \mathrm{~g})$ weekly for 4 weeks. The rats were immobilized on the sample plate of a stereoscopic microscope (AKS- IIILF, OMAX, St. Maesil, Sejong, Korea), and $25 \mu \mathrm{L}$ sodium hydronate [Hyaluronic acid eye (18 mg/1.2 mL), Kukje Pharma, St. Yatap, Sungnam, Korea] was injected into the right eye using a Hamilton syringe (Gastight \#1705, Hamilton Co., Reno, NV, USA) and a 30-gauge needle directed through the corneal limbus (Moreno et al. 2005).

\section{IOP assessments using two types of tonometers}

After injecting hyaluronic acid intracamerally, eye drops containing $0.5 \%$ proparacaine hydrochloride (Alcaine ${ }^{\circledR}$, Alcon Inc., Fort Worth, TX, USA) were used as topical anaesthesia. IOPs were measured using a rebound tonometer (Tonolab, Tiolat, OY, Helsinki, Finland) and an immersive tonometer (Tonopen ${ }^{\circledR} \mathrm{XL}$, Medtronic Wolan, Jacksonville, FL) at about 3:00 pm time of day. The measurements were obtained by a skilled researcher who fixed the neck and body of the rat with one hand, not allowing the rat to move (Kim et al. 2014).

Ten eyes of IOPs ( 5 glaucomatous right eyes and 5 normotensive left eyes) were measured using the Tonolab. The probe was positioned perpendicular to the cornea and horizontal to the ground such that there was 1-4 mm between the tip of the probe and the cornea ( $\mathrm{Yu}$ et al. 2007). After six measurements had been obtained, the mean IOP was shown in the display window. If an error message appeared at any time, the measurement was

Table 1. Comparison of intraocular pressure (IOP) obtained by Tonopen XL vs Tonolab in normotensive and glaucomatous eyes*.

\begin{tabular}{llll}
\hline Parameters & $\begin{array}{l}\text { Tonopen XL } \\
(\mathbf{m m H g})\end{array}$ & $\begin{array}{l}\text { Tonolab } \\
(\mathbf{m m H g})\end{array}$ & p value \\
\hline $\begin{array}{l}\text { Normotensive eyes } \\
\text { Left eyes (n=10) }\end{array}$ & $10.80 \pm 1.03$ & $15.10 \pm 0.73$ & $\mathrm{p}=0.1$ \\
$\begin{array}{l}\text { Glaucomatous eyes } \\
\text { Right eyes (n=10) }\end{array}$ & & \\
\hline
\end{tabular}

*Paired t-test. repeated. The rest ten eyes of IOPs (5 glaucomatous right eyes and 5 normotensive left eyes) were evaluated using the Tonopen. The Tonopen was attached to the apex of the central cornea. Three times of IOP measurements per eye with variances of less than $5 \%$ on each measurement were obtained and averaged for determination of IOP.Statistical analysis was performed using SPSS (version 24.0; SPSS Inc., Chicago, IL).

\section{Haematoxylin staining of control and experimental} eyes (especially retina)

Haematoxylin staining was performed to confirm that the glaucoma rat model was well established. After measuring IOP, enucleation was performed on postoperative week 4 . The eyeballs were soaked in $4 \%$ paraformaldehyde ( $\mathrm{pH}$ 7.4) and sectioned along the posterior margin of the ora serrata. The retina was peeled off with a number 0 brush and the retinal tissue was divided into four quadrants. The mid-papillary regions of the retinas were then cut into small rectangular shaped sections. The retinal tissues were fixed in $4 \%$ paraformaldehyde for 2 hours at $4{ }^{\circ} \mathrm{C}$. After fixation, they were transferred to a $30 \%$ sucrose solution (pH 7.4) and refrigerated overnight. The retinal tissues were flash frozen in liquid nitrogen and stored at $-70^{\circ} \mathrm{C}$. Finally, they were rinsed in phosphate buffered saline (PBS) $(\mathrm{pH}$ 7.4), dehydrated, embedded in wax, and cut into $5 \mu \mathrm{m}$ sections and finally stained with haematoxylin and eosin.

All retinal preparations were evaluated by microscope (Nikon ECLIPSE 80i, Nikon Corporation, Japan) and were captured with I-Solution ${ }^{\mathrm{TM}}$ (IMT I-Solution Inc., Vancouver, BC, Canada).

\section{RESULTS AND DISCUSSION}

\section{IOP values using two types of tonometers}

The rat glaucoma model was induced by elevated IOP within 1 hour of intracameral injection. The mean IOP values of normotensive eyes (left eyes) were $10.80 \pm 1.03$ $\mathrm{mmHg}$ by Tonopen, and $15.10 \pm 0.73 \mathrm{mmHg}$ by Tonolab. The IOP values were statistically insignificant each other $(\mathrm{p}=0.1$, independent paired $\mathrm{t}$-test $)$. The mean IOP values of glaucomatous eyes (right eyes) were $30.20 \pm 2.67$ $\mathrm{mmHg}$ by Tonopen, and $37.90 \pm 2.73 \mathrm{mmHg}$ by Tonolab. They were statistically insignificant $(\mathrm{p}=0.95$, independent paired t-test) (Table 1).

The Spearman correlation coefficient between the IOP values of the normotensive eyes using two tonometers was $0.273(\mathrm{p}=0.445)$ which indicated negative correlation. The Spearman correlation coefficient between the IOP values of the glaucomatous eyes was 0.904 ( $p<0.01$ ) indicating strong positive correlation with 


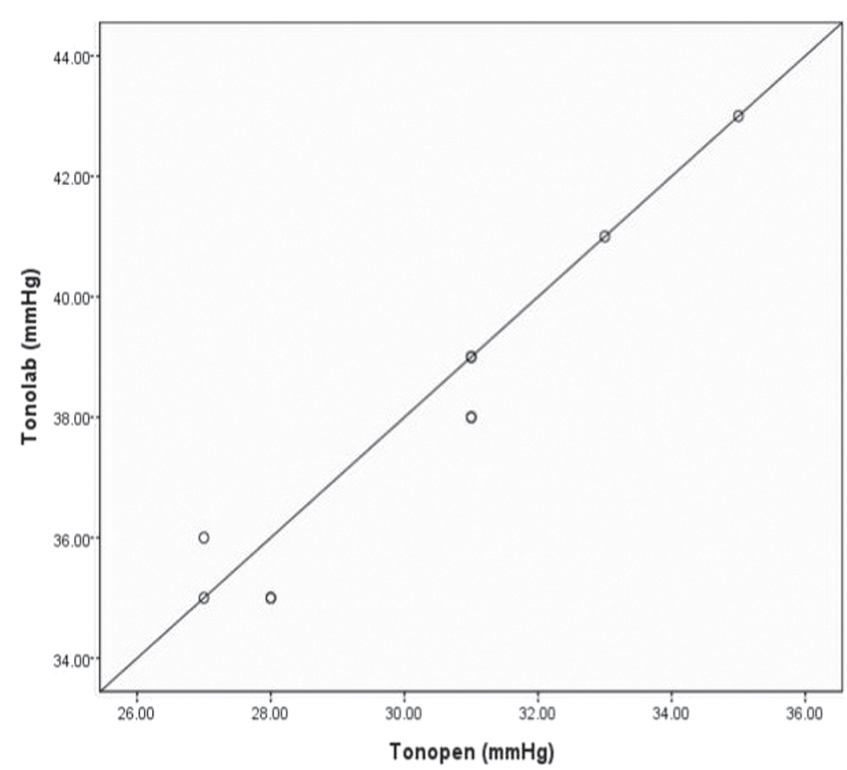

Fig. 1. Scatter plot showing measurement values with Tonopen XL and Tonolab. There is reasonably good linear correlation coefficient between the IOP values of the glaucomatous eyes, i.e., 0.904 ( $p<0.01)$.

each other. In glaucomatous eyes, the regression equation between Tonopen and Tonolab were $\mathrm{y}=\mathrm{x}+8$ (Fig. 1).

\section{Histological changes of normotensive and glaucomatous eyes}

There was significant loss of ganglion cell layer (GCL) cells in retina of glaucomatous eye compared to that of normotensive eye (Fig. 2A, B). Diminution of ganglion cell layer (GCL) cells in glaucomatous eye was observed whereas the other retinal layers showed normal appearance.

Measurement of IOP is essential to make accurate diagnosis on ophthalmic diseases, such as glaucoma and uveitis. Among the risk factors for glaucoma, IOP is the only major risk factor that can be controlled.

Not only people, but the number of Veterinary Ophthalmologists is also currently increasing in Korea and Japan due to the increase in adoption of pets (Nagata et al. 2011). Our glaucoma model induced by intracameral injection of hyaluronic acid was reported by Moreno et al. (2005). Intracameral injection of hyaluronic acid is the fastest way to increase IOP in short period. Because of cohesive characteristics of hyaluronic acid, the direct flow of aqueous humor from the ciliary process to the pupil is blocked, and high IOP is developed. Previously, experimental glaucoma models in rats resulting from chronic elevations in IOP were created through intravitreal endothelin-1 injection (Dibas et al. 2008), episcleral vein injection with hypertonic saline (Johnson et al. 2007, Guo et al. 2011) and cauterizing of episcleral veins (Bai et al. 2014). This is the first study to compare the IOP of rats with this type of glaucoma model.

There are many kinds of tonometers to measure IOP in animal model. Since making glaucoma model through intracameral injection of hyaluronic acid is invasive, we chose two most commonly used noninvasive tonometers that can be used to measure IOP of rats. Tonopen and Tonolab are non-invasive methods to estimate of IOP (Millar and Pang 2015). To reduce the likelihood that the test results would be affected by laboratory environment and animal conditions, we used the same age group of rats and measured at 3:00 pm time to avoid the effects of diurnal fluctuations in IOP (Rajaei et al. 2017).

There was no statistically significant difference between the normotensive eyes and glaucomatous eyes measured by the two types of tonometers. However, the IOP measured by Tonolab tended to be higher than the IOP measured by Tonopen. Nagata et al. (2011) reported that IOP measurements of Beagle dogs also had no significant difference in the accuracy of IOP evaluated by rebound tonometer and Tonopen. They argued that tonopen was less accurate than Tonolab in the hypertensive pressure stage for over $20 \mathrm{mmHg}$.

However, in our study, the IOP measured by Tonopen and Tonolab in the control group was negatively correlated. Tonopen is an immersive tonometer used to measure the IOP of several animals as well as rats (Mermoud et al. 1994, Midelfart and Wigers 1994). The probe is relatively large in the rat eye and the examiner must press the center of the cornea to measure IOP. As a result, the measured IOP depends on the degree of succession of the examiner and varies greatly depending on the examiner. There is a disadvantage in that even when one examiner measures, there is a large deviation in each measurement (Moore et al. 1993). Tonolab is a rebound tonometer based on the inductive or impact principle (Kontiola 1996). This tonometer is a device that measures the electromagnetic force generated by the momentum when the small magnetic probe is shot on the cornea and then bounced back and converts it into the intraocular pressure (Kontiola 1996). For these reasons, there were statistically insignificant differences in the IOP measured with two tonometers. In this study, Tonolab was measured first in the order of IOP measurement, so it can be considered that the first measured IOP can have certain influence on the later IOP. However, unlike Tonopen, the probe of Tonolab is very small and lightweight, so its effect is less due to the small force applied to the cornea at the time of contact. 

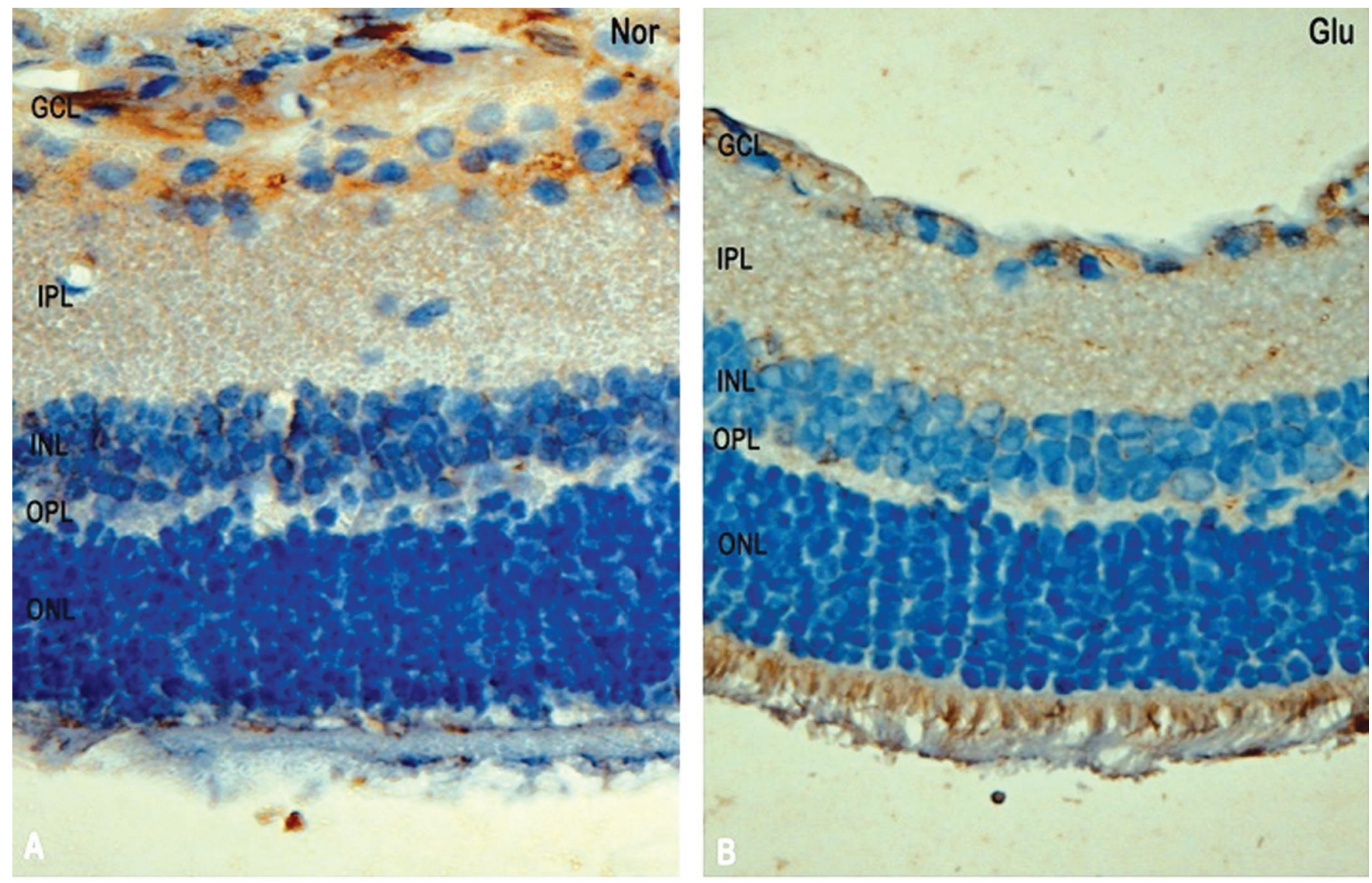

Fig. 2. Light micrographs of transverse sections of retinas from contralateral eye (A) and glaucomatous eye which was injected with hyaluronic acid (B). [Nor = ormotensive eyes; Glu = glaucomatous eyes; IPL = inner plexiform layer; INL = inner nuclear layer; $\mathrm{OPL}=$ outer plexiform layer; ONL = outer nuclear layer. Haematoxylin stain (magnification , X400).]

The limitations of this study were, firstly, a short-term experiment with a small number of mice. The number of cases was small and the measured IOP values by Tonopen were highly variable. It may appear that there is no correlation at normal IOP. Further studies will be needed to develop a new model for a large number of rats in the future. The second one was that we measured the IOP only one day. The glaucoma with high IOP was maintained well on the day of injecting the hyaluronic acid and only the day of the procedure was measured because the viscoelastic material escaped from the injection site with time.

\section{CONCLUSION}

Tonopen and Tonolab measurements showed a significant positive correlation within high IOP. In conclusion, Tonopen should be used carefully when the IOP is in normal range, and both Tonopen and Tonolab can be used when the IOP is high.

\section{ACKNOWLEDGEMENT}

The authors are thankful to Professor Jae-Ho Shin, PhD and Professor Mun-Yong Lee, MD, PhD.

\section{REFERENCES}

Anders F, Mann C, Liu A, Teister J, Funke S et al. (2018) Correlation of crystallin expression and RGC susceptibility in experimental glaucoma rats of different ages. Curr Eye Res 43(10): 1267-1273.

Bai Y, Zhu Y, Chen Q, Xu J, Sarunic MV et al. (2014) Validation of glaucoma-like features in the rat episcleral vein cauterization model. Chin Med J (Engl) 127(2): 359-364.

Choy YJ, Shin JH, Seok PR, Choi JH (2017) Expression of slit2 and robo Receptors in high tension glaucoma: a rat glaucoma model. Annals Optometry Contact Lens 16(1): 1016.

Dibas A, Yang MH, He S, Bobich J, Yorio T (2008) Changes in ocular aquaporin-4 (AQP4) expression following retinal injury. Mol Vis 14: 1770-1783. 
Comparison of intraocular pressure in Spargue-Dawley rats...

Guo Y, Johnson EC, Cepurna WO, Dyck JA, Doser T et al. (2011) Early gene expression changes in the retinal ganglion cell layer of a rat glaucoma model. Invest Ophthalmol Vis Sci 52(3): 1460-1473.

Johnson EC, Jia L, Cepurna WO, Doser TA, Morrison JC (2007) Global changes in optic nerve head gene expression after exposure to elevated intraocular pressure in a rat glaucoma model. Invest Ophthalmol Vis Sci 48(7): 3161-3177.

Kim YR, Kang WS, Kweon EY, Cho NC, Lee DW (2014) Steroid-induced ocular hypertension model in the mice. Korean J Ophthalmol 55(8): 1202-1207.

Kontiola A (1996) A new electromechanical method for measuring intraocular pressure. Doc Ophthalmol 93(3): 265276.

Mermoud A, Baerveldt G, Minckler DS, Lee MB, Rao NA (1994) Intraocular pressure in Lewis rats. Invest Ophthalmol Vis Sci 35(5): 2455-2460.

Midelfart A, Wigers A (1994) Clinical comparison of the Proton and Tono pen tonometers with the Goldmann applanation tonometer. Br J Ophthalmol 78(12): 895-898.

Millar JC, Pang IH (2015) Non-continuous measurement of intraocular pressure in laboratory animals. Exp Eye Res 141: 74-90.

Moore CG, Milne ST, Morrison JC (1993) Noninvasive measurement of rat intraocular pressure with the Tono pen. Invest Ophthalmol Vis Sci 34(2): 363-369.
Moreno MC, Marcos HJ, Croxatto JO, Sande PH, Campanelli J et al. (2005) A new experimental model of glaucoma in rats through intracameral injections of hyaluronic acid. Exp Eye Res 81(1): 71-80.

Morrison JC, Moore CG, Deppmeier LM, Gold BG, Meshul CK et al. (1997) A rat model of chronic pressure-induced optic nerve damage. Exp Eye Res 64(1): 85-96.

Nagata N, Yuki M, Hasegawa T (2011) In vitro and in vivo comparison of applanation tonometry and rebound tonometry in dogs. J Vet Med Sci 73(12): 1585-1589.

Quigley HA, Addicks EM (1980) Chronic experimental glaucoma in primates. I. Production of elevated intraocular pressure by anterior chamber injection of autologous ghost red blood cells. Invest Ophthalmol Vis Sci 19(2): 126-136.

Quigley HA, Hohman RM (1983) Laser energy levels for trabecular meshwork damage in the primate eye. Invest Ophthalmol Vis Sci 24(9): 1305-1307.

Rajaei SM, Mood MA, Paryani MR, Williams DL (2017) Effects of diurnal variation and anesthetic agents on intraocular pressure in Syrian hamsters (Mesocricetus auratus). Am J Vet Res 78(1): 85-89.

Yu YC, Kim SH, Kim YJ, Park KH (2007) Comparison of the intraocular pressure measurement between rebound tonometer and tonopen in rats. Korean J Ophthalmol 48(1): 135-141.

Cite this article as: Choy YJ, Choi JH (2021) Comparison of intraocular pressure in Spargue-Dawley rats using two types of tonometers. Explor Anim Med Res 11(2): 152-156. DOI : 10.52635/eamr/11.2.152-156. 